\title{
Application of Split Ring Resonator (SRR) Loaded Transmission Lines to the Design of Angular Displacement and Velocity Sensors for Space Applications
}

\author{
Javier Mata-Contreras, Cristian Herrojo, Student Member IEEE, and Ferran Martín, Fellow IEEE
}

\begin{abstract}
The accurate measurement of the angular displacement and velocity of reaction wheels is necessary for attitude (orientation) control in space vehicles (satellites). In this paper, microwave, contactless and low cost (as compared to optical encoders) sensors useful for that purpose are analyzed in detail. The sensor consists of a rotor and a stator. The rotor is a disk (or a circular crown) of dielectric material, where one or several arrays of equidistant single-loop split ring resonators (SRRs) are etched along its edge, forming circular chains of hundreds of SRRs. The stator is a coplanar waveguide (CPW) also loaded with pairs of single-loop SRRs (etched in the back substrate side), with the centers located in the slot region. The sensing principle is based on the amplitude modulation of a harmonic (single tone continuous wave) feeding signal, achieved when the chains of the rotor are displaced over the SRR pairs of the stator. Both sensor elements (rotor and stator) must be parallel oriented, with the SRR pairs of the CPW in close proximity to the SRR chains of the rotor (and rotated $180^{\circ}$ ), in order to favor their coupling. By this means, the transmission coefficient of the CPW is varied by the circular motion of the rotor, and significant amplitude modulation of the feeding signal is achieved. From the envelope function, the angular velocity can be accurately determined. With the proposed sensors, instantaneous and practically unlimited rotation speeds can be measured.
\end{abstract}

Index Terms- Metamaterials, split ring resonators (SRRs), microwave sensors, angular velocity sensors.

\section{INTRODUCTION}

$\mathrm{M}$ ETAMATERIAL transmission lines based on electrically small resonators (split ring resonators SRRs [1], [2]- and complementary split ring resonators CSRRs [3], [4]- among others) have been demonstrated to be useful in many applications. On the basis of impedance and dispersion engineering [5], enhanced bandwidth devices [6], multiband components [7], [8], or leaky wave antennas with backward-to-forward scanning capability [9], have been designed. In other applications, the resonance phenomenon is the key aspect to explain device functionality. Such applications include filters [10], [11], multiband dipole and monopole antennas [12], [13],

Manuscript received December 22, 2016; revised February 21, 2017 and March 28, 2017; accepted April 6, 2017. This work was supported by MINECO-Spain (projects TEC2013-40600-R and TEC2016-75650-R), by Generalitat de Catalunya (project 2014SGR-157), by the European Space Agency (ESA), through project 4000111799/14/NL/SC coordinated by EMXYS Embedded Instruments, by Institució Catalana de Recerca Estudis Avançats (who awarded Ferran Martín), and by FEDER funds.

J. Mata Contreras, C Herrojo and F. Martín are with GEMMA/CIMITEC, Departament d'Enginyeria Electrònica, Universita Autònoma de Barcelona, 08193 Bellaterra, Spain. E-mail: Ferran.Martin@uab.es. radiofrequency barcodes [14], and microwave sensors [15], [16].

Concerning sensors, the subject of interest in this work, several strategies or principles have been reported for sensing in resonator-loaded lines, including variation of the notch frequency (or frequencies) [15], [17]-[25], frequency splitting [26]-[28], or modulation of the notch magnitude [16], [29]-[37]. Many different variables can be measured through the aforementioned sensing principles, but these sensors implemented through transmission lines with metamaterial loading are especially useful for measuring spatial variables, such as linear or angular displacement and velocities.

Recently the authors have reported angular displacement and velocity sensors implemented by means of circularly shaped coplanar waveguides (CPWs) [35] or microstrip lines [36], loaded with electric LC (ELC) resonators (first reported in [38]), or with S-shaped SRRs (S-SRR) [37]. In these implementations, the resonant element, etched on a movable microwave substrate, is axial to the rotating object, and the transmission line is placed beneath the rotating resonator, parallel to it, and with the line axis intersecting the axis of the resonator (Fig. 1).

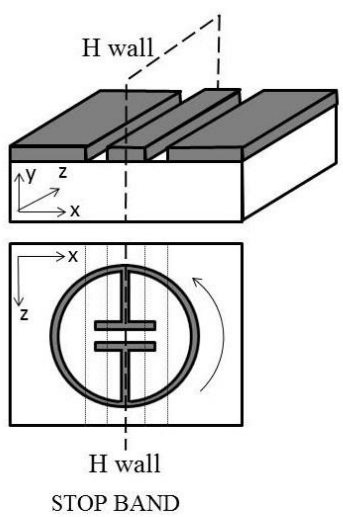

(a)

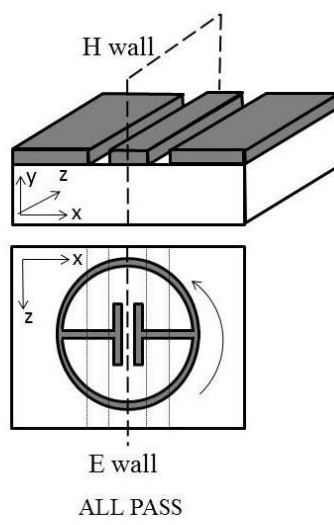

(b)
Fig. 1. Relative position between CPW and the ELC resonator: (a) Orientation with maximum coupling; (b) Orientation with minimum coupling.

Under these conditions, the magnetic coupling between the line and the resonant element (ELC or S-SRR) is controlled by the relative orientation (angle) between line and the resonator, and both the angular displacement and velocity can be measured. Indeed, there are two extreme orientation conditions corresponding to maximum and minimum coupling (see Fig. 1). In (b), neither the magnetic field nor the electric field generated by the line are able to excite the ELC at its fundamental resonance since the magnetic wall (H-wall) of the line and the electric wall (E-wall) of the 
resonator are perfectly aligned. For any other angular orientation, coupling arises, this being maximum for the orientation indicated in (a), where the $\mathrm{H}$-wall of line and resonator are aligned.

With minimum coupling, the line is transparent, whereas for maximum coupling a strong notch at the resonance frequency of the resonant element arises. In this rotation sensor, the angular displacement is determined by the notch magnitude. The angular velocity is derived from the distance between adjacent peaks in the envelope function of the amplitude modulated signal that results by feeding the line by a harmonic (single tone continuous wave) signal tuned at the resonance frequency of the ELC or S-SRR [35]-[37].

In the previous axial rotation sensor, practically unlimited angular velocities can be measured with high accuracy (note that the frequency of the carrier signal is orders of magnitude higher than the angular frequency of the rotor, for any reasonable rotating system). However, instantaneous velocities cannot be measured with this system, since the peaks in the envelope function appear twice per cycle. In order to measure instantaneous velocities, able to vary within a cycle, it is necessary to achieve as many peaks in the envelope function as possible. The solution is the edge configuration, first proposed in [39], where a chain of resonators (SRRs) was etched along the edge of a disk (or circular crown) made of dielectric material, attached axially to the rotor (Fig. 2). By placing the stator, a CPW transmission line also loaded with a pair of SRRs (for the reasons that will be explained later), in front of the SRR chain, as many peaks as resonant elements of the chain arose, opening the possibility to accurately measure potential variations of the angular velocity within a cycle.

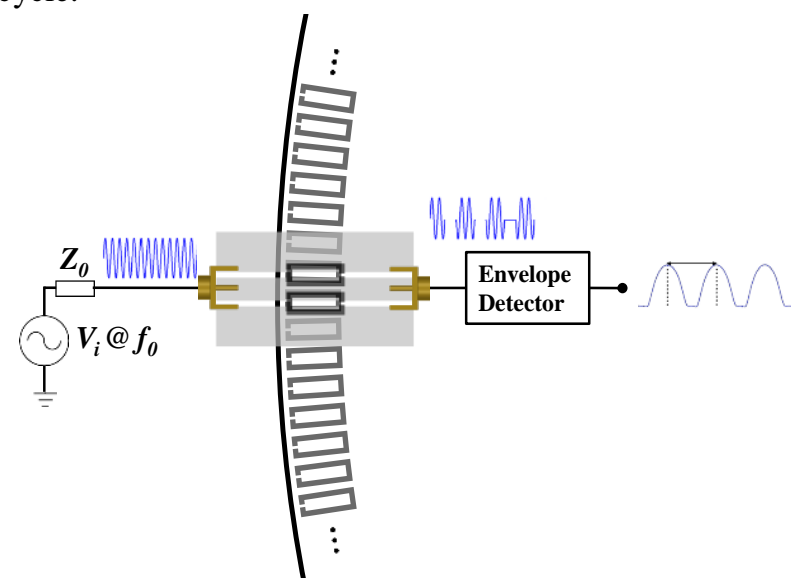

Fig. 2. Sketch of the rotation sensor based on the edge configuration.

Other angular displacement and velocity sensors are based on a radio link between two circularly polarized (CP) antennas [40]. If one of these antennas is rotated, the link transfer function is changed and this effect is proposed for the measurement of angular velocity. This strategy has been proposed for the measurement of small changes in angular velocity or angular displacement, not easily achievable in sensors based on pulses (as those reported in [35]-[37] by the authors, or in [41]-[43]). However, the sensors proposed in this paper contain one or more resonator chains with a high number of resonant elements. Hence instantaneous velocities can indeed be measured, due to the large number of generated pulses per cycle, as will be demonstrated. As compared to the work [39], in this paper we modify the sensing strategy by considering a different criterion to set the frequency of the harmonic feeding signal of the CPW, with the objective to double the number of pulses per cycle. Moreover, angular velocity sensors with two chains of resonant elements, useful to further increase the number of pulses per cycle, are also presented. The paper includes the analysis of the effects of air gap separation between the SRRs of the rotor and stator, an important parameter with significant influence on sensor performance.

The paper is organized as follows. The sensing principle is explained in detail in Section II, where the specific strategy to set the frequency of the harmonic feeding signal and the benefits of considering two chains of SRRs are pointed out. The effects of the air gap are discussed in Section III. The sensing system, including the RF module and the envelope detector electronics is presented in Section IV. The sensor functionality is demonstrated in this section, where angular velocity measurements, considering the two designed rotors (with one or two SRR chains) and stators (with one or two pairs of SRRs, as well), are carried out. Finally, the main conclusions are highlighted in Section V.

\section{SENSING PRINCIPLE}

\section{A. Rotor with a Single Chain of SRRs}

To discuss the sensing principle of the proposed angular velocity sensors, let us first consider, without loss of generality, the case of a single circular chain of SRRs etched in the rotor. Consequently, the stator is implemented by means of a coplanar waveguide (CPW) transmission line loaded with a single pair of SRRs. The cross sectional view of the sensor layers and the topologies of the stator and rotor (zoom view) are depicted in Fig. 3. Note that although the SRRs of the rotor are disposed in a circular chain, their geometry is rectangular. Since the radius of the rotor is much larger than SRR dimensions, it can be considered that the space between SRRs is uniform and that they can form perfectly broadside geometries with the SRRs of the stator.

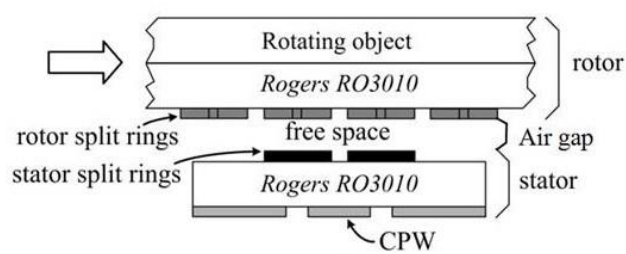

(a)

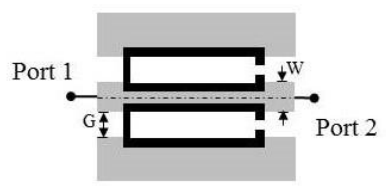

(b)

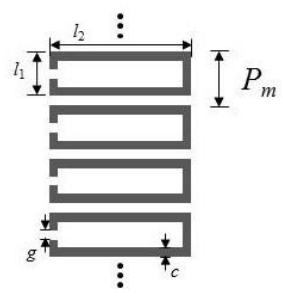

(c)
Fig. 3. Geometry of the proposed sensor realized on a dielectric substrate with relative permittivity of 11.2 and loss tangent of 0.0023 . Dimensions (in $\mathrm{mm}$ ) are: substrate thickness of $0.635 \mathrm{~mm}$ for the stator and $1.27 \mathrm{~mm}$ for the rotor, $W=1.3, G=0.9, P_{m}=2, l_{1}=1.6, l_{2}=6.2, c=0.4$, and $g=0.2$. (a) Sensor cross-section; (b) layout of the stator; (c) layout of rotor section 
As can be seen in Figs. 3(b) and (c), the split rings of the rotor are identical to those in the stator, but rotated $180^{\circ}$. Let us now justify the need to include an identical pair of rotated SRRs in the stator part. Indeed, if the pair of SRRs of the stator is removed, coupling between the CPW transmission line and the pair of rings of the rotor closer to the slot regions of the $\mathrm{CPW}$ arises. However, since the rings of the rotor must be closely spaced (in order to accommodate a large number of resonators and, hence, pulses per cycle), coupling between neighbouring SRRs of the rotor [44], and between the CPW and additional SRRs of the rotor (not only those overlapping the slot regions) is inevitable. These extra couplings degrade the sensor performance and must be avoided. To this end, a pair of SRRs is etched in the back side of the CPW transmission line, as indicated in Fig. 3 [39].

Let us assume that a pair of SRRs in the rotor is perfectly aligned (face to face) with the SRR pair of the stator. Under these conditions, the two pairs of SRRs are coupled to the line, but such coupling occurs at the resonance frequency of the vertical (coupled) pairs, which is substantially different than the resonance frequency of a single split ring. Such vertically coupled SRRs, with rings rotated $180^{\circ}$, constitute the so-called broadside coupled split ring resonator (BCSRR) [45], [46]. The relative $180^{\circ}$ orientation between the SRRs of rotor and stator is necessary to enhance their coupling. The key advantage of this configuration is the fact that since coupling between the line and the BC-SRRs occurs at a frequency substantially lower than the frequency of the single SRRs, the above cited extra couplings are cancelled by tuning the harmonic feeding signal at the resonance frequency of the BC-SRRs. This was the strategy used in [39] for the implementation of the angular velocity sensors. The insertion loss of the CPW with the SRRs of the rotor perfectly aligned with those of the stator (forming a perfectly aligned BC-SRR) is depicted in Fig. 4. Besides the response for this relative position between the rotor and the stator (called reference, $R E F$, position), with perfectly aligned SRRs, the figure includes the response for other relative incremental/decremental displacements (corresponding to misalignments), expressed in terms of the period of the rotor, $P_{m}$.

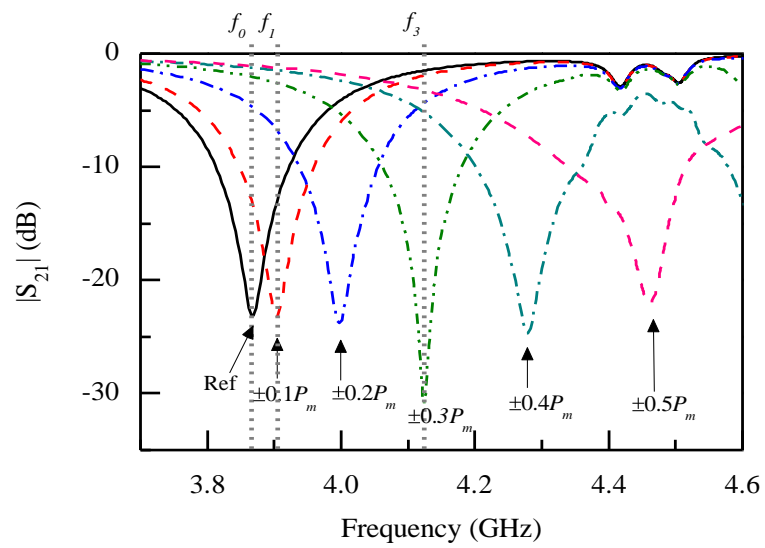

Fig. 4. Electromagnetic simulation, using Keysight Momentum, of the transmission coefficient for different relative displacements between the stator and the rotor (air gap of $0.5 \mathrm{~mm}$ )

In Fig. 4 , it can be appreciated that the attenuation at $f_{0}$, the frequency of the perfectly aligned BC-SRRs, strongly depends on the relative displacement between the rotor and the stator. However, there is not a firm reason, a priori, to tune the feeding signal at such frequency. Indeed, if we chose a frequency different from $f_{0}$, two attenuation peaks, rather than one, are expected as the rotor moves one period (between $-P_{m} / 2$ and $+P_{m} / 2$ ) above the stator. As closer the selected frequency to $f_{0}$ is, the smaller the separation between attenuation peaks becomes. This effect is illustrated in Fig. 5, where the insertion loss for different frequencies, as the rotor is displaced above the stator, is depicted. Note also that as the two attenuation peaks approximate (by driving the frequency towards $f_{0}$ ) the transmission level between such peaks decreases, and the peaks merge when the considered frequency is $f_{0}$. The main conclusion of this study is that in order to increase the number of pulses per cycle, it is convenient to feed the CPW transmission line with a carrier frequency $f_{c}$ satisfying $f_{c} \neq f_{0}$. The specific frequency value should be chosen in order to obtain similar transmission between attenuation peaks (e.g., $f_{c} \approx f_{3}$ is a good choice, according to Fig. 5). By injecting a harmonic signal tuned at that frequency, the rotor motion will modulate the amplitude at the output port of the CPW transmission line. From the envelope, variations in the instantaneous rotation speed can be inferred with as much precision as higher the number of pulses per cycle is. With the proposed strategy, the number of pulses per cycle is twice the number of pulses achieved in [39].

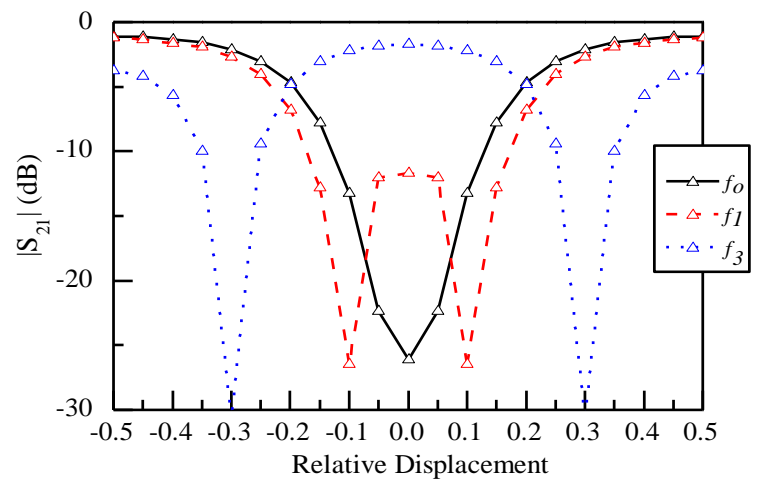

Fig. 5. Attenuation as a function of the rotor relative displacement (in terms of $\mathrm{P}_{\mathrm{m}}$ ) at the indicated frequencies.

As has been indicated, the resonance frequency shifts upwards as the rings are misaligned (Fig. 4). However, for strong misalignment, the resonance peaks are degraded due to the parasitic (unwanted) effects mentioned before (rotor inter-resonator coupling and multiple SRR-to-CPW coupling). This means that if the frequency of the harmonic feeding signal is chosen close to the one corresponding to the resonance for $\pm 0.5 P_{m}$ misalignment, the modulation index will be degraded, and this is an unwanted effect.

The most important benefit of the proposed approach is the fact that inter-resonator spacing in the rotor can be driven to the limits dictated by the fabrication technology. Nevertheless, the number of resonators that can be accommodated along the rotor perimeter is limited by their dimensions as well. The size of the resonant elements is inversely proportional to the frequency of the feeding signal. By increasing that frequency, SRR dimensions are 
reduced. However, depending on the specific application, such frequency may not be a design variable.

To end this sub-section, let us show the current distributions for two different rotor positions (misalignments). The results are plotted in Fig. 6. Such current distributions have been obtained at the resonance frequency of the corresponding misalignments. As can be seen, the excited SRRs of the rotor are those that are totally (case of perfect alignment, resonance frequency $3.87 \mathrm{GHz}$ ) or partially (misalignment, resonance frequency $4.29 \mathrm{GHz}$ ) intersecting the SRRs of the stator, as expected.

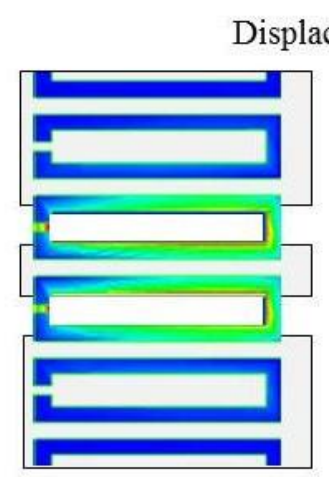

$\mathrm{f}=3.87 \mathrm{GHz}$

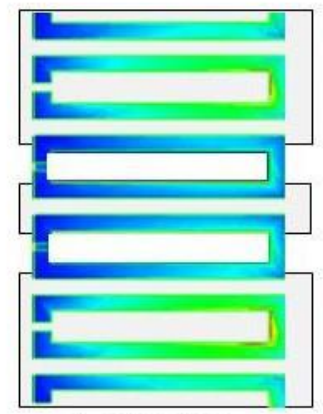

$\mathrm{f}=4.29 \mathrm{GHz}$

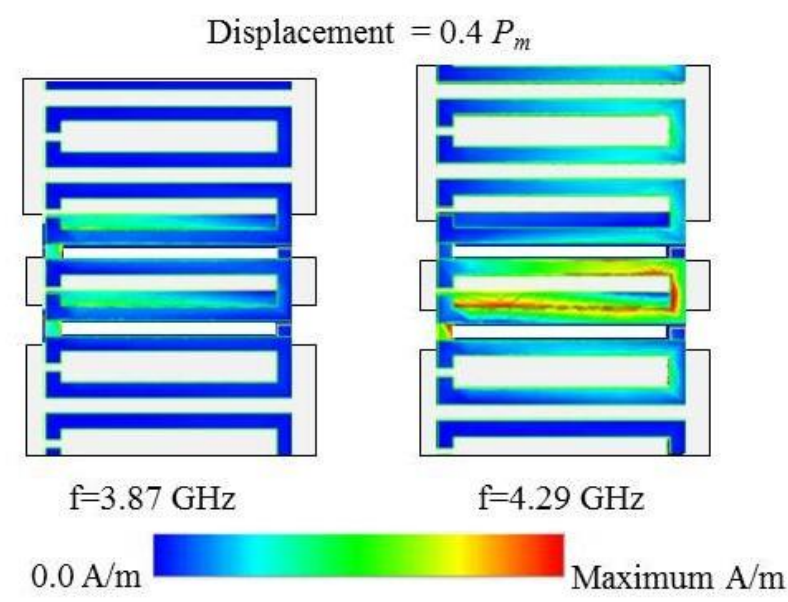

Fig. 6. Current distributions for different misalignments inferred from Keysight Momentum. The considered frequencies are also indicated.

\section{B. Rotor with Two Chains of SRRs}

To increase the number of pulses per revolution, an alternative approach is to consider two circular SRR chains in the rotor and two pairs of split rings in the stator, as depicted in Fig. 7. The two SRR chains are offset by a distance $\mathrm{P}_{\mathrm{m}} / 2$, which is half the width between SRRs.

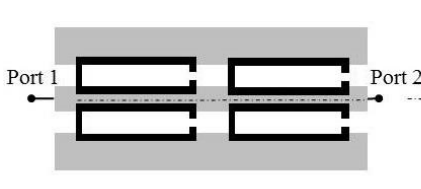

(a)

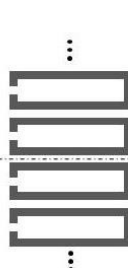

(b)

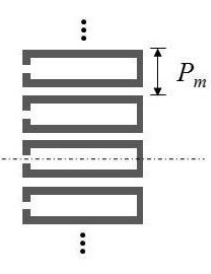

Fig. 7. (a) Layout of the stator; (b) layout of the rotor section, for the case of rotor with two SRR chains.
Figure 8 depicts the response (insertion loss) of the CPW transmission line (stator) for different relative displacements between stator and rotor. The behavior is similar to the one of Fig. 4, in reference to the case with a single SRR chain. Except for the curve corresponding to $\mathrm{Pm} / 4$ displacement, two slightly different responses result for the different displacements between the rotor and stator. The reason is that, in the real rotor, the two chains of SRRs are not exactly identical.

A rich phenomenology, to be discussed next, arises by the presence of two SRR chains. First of all, if the frequency of the feeding signal is the resonance frequency of the perfectly aligned BC-SRR, $f_{0}$, two attenuation peaks, rather than one, appear when the rotor experiences a relative motion between $-P_{m} / 2$ and $+P_{m} / 2$ (see Fig. 9). The reason is that there are two positions within this range where either the inner or the outer SRR chain forms a perfectly aligned BC-SRR with one of the SRRs of the stator. For frequencies different from $f_{0}$, four attenuation peaks, rather than two, are expected as the rotor moves within one SRR chain period, as can be seen in Fig. 9. Indeed, each SRR chain acts independently, and for that reason the number of attenuation peaks is twice the one corresponding to a single chain of SRRs. There is, however, a special case. Namely, if the frequency of the feeding signal is chosen as the one corresponding to the resonance for a relative displacement between the stator and the rotor of exactly $\pm P_{m} / 4$ (see the insertion loss for that case in Fig. 8), then only two attenuation peaks appear (see Fig. 9). For this singular misalignment $\left( \pm P_{m} / 4\right.$, see Fig. 10$)$, the two SRR chains exhibit an undistinguishable relative displacement with regard to the corresponding SRR-pair of the stator. This is the reason that explains the presence of only two attenuation peaks in this case. However, note that the depth of the attenuation peaks is stronger since both chains act simultaneously. This effect is also visible in Fig. 8, where the resonance peak for this misalignment is clearly more pronounced. Let us call $f_{s}$ the resonance frequency for this latter case. If the feeding signal is either tuned at $f_{0}$ or $f_{s}$, two pulses per period appear; otherwise, four attenuation peaks (or pulses) arise. Nevertheless, depending on the specific feeding frequency, i.e., if it is close to $f_{0}$ or $f_{s}$, it is possible that the four peaks are not perfectly visible.

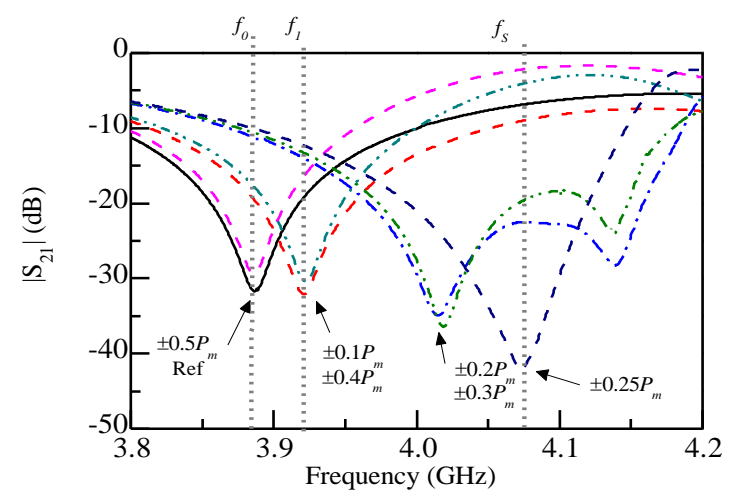

Fig. 8. Electromagnetic simulation, using Keysight Momentum, of the transmission coefficient for different relative displacements between the stator and the rotor for the rotor with two SRR chains. 


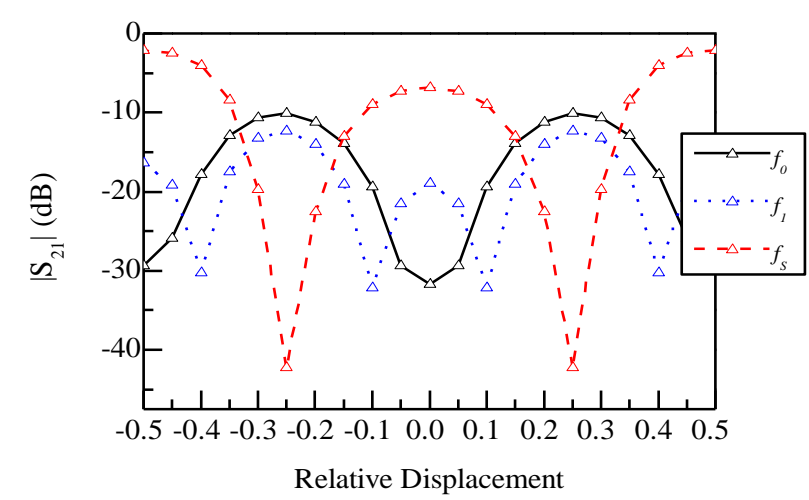

Fig. 9. Attenuation as a function of the rotor displacement at the indicated frequencies for the rotor with two SRR chains.

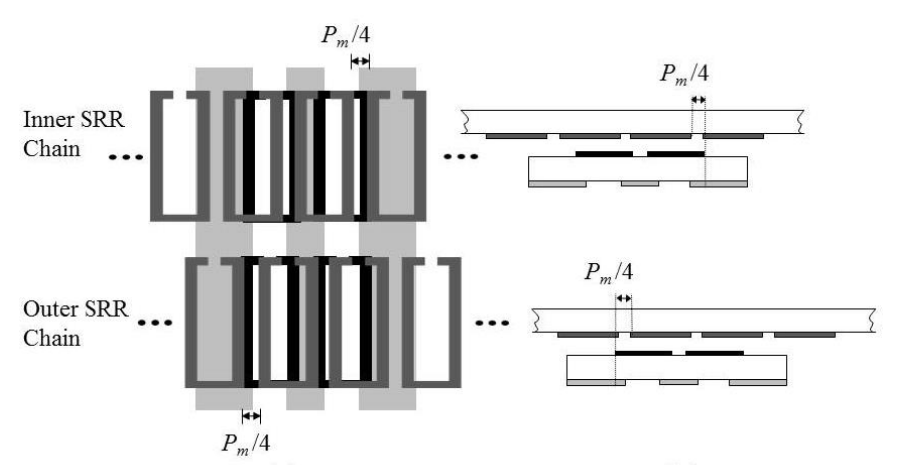

(a)

(b)

Fig. 10. View of the stator/rotor configuration for the particular case of $\pm P_{m} / 4$ misalignment. (a) Top view; (b) cross-section.

\section{EFFECTS OF THE AIR GAP}

Since the resonances that appear in Figs. 4 and 8 are due to the combined effect of the split rings of the stator and rotor, it follows that such resonances should be influenced by the air gap separation between them. Figure 11 presents the insertion loss of the CPW for different relative displacements between the stator and the rotor parameterized by the air gap separation. The figure corresponds to the case of a single chain of SRRs for simplicity. Note that such relative displacements correspond to variation between $0 \mathrm{P}_{\mathrm{m}}$ and $\mathrm{P}_{\mathrm{m}} / 2$ in steps of $0.05 \mathrm{P}_{\mathrm{m}}$. The curves corresponding to $\mathrm{P}_{\mathrm{m}} / 2$ are indicated by dashed lines.

As the air gap increases, the frequency range where resonances appear (for different relative displacements) progressively decreases. Note, however, that this constriction is due to the increase of the BC-SRR resonance frequency (the smallest one). The resonance frequency corresponding to a semi-period displacement $\left( \pm 0.5 P_{m}\right)$ does not experience a significant variation. The reason is that the resonance frequency for such strong misalignment is mainly dictated by the pair of rings of the stator (i.e., the coupling with the rings of the rotor is negligible), and hence the air gap distance has insignificant effect. This narrowing effect is shown in Fig. 12, where the limits of the resonances range as a function of the air gap distance is represented.
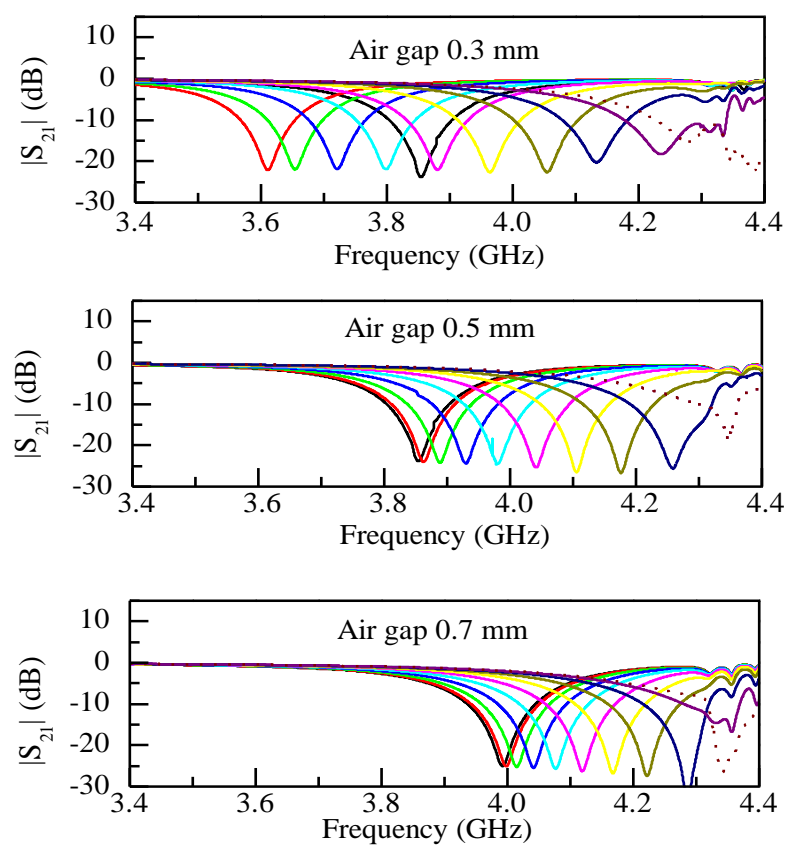

Fig. 11. Electromagnetic simulation, using Keysight Momentum, of the transmission coefficient for different relative displacements (from $0 \mathrm{~mm}$ to $1 \mathrm{~mm}$ in steps of $0.1 \mathrm{~mm}$ ) between the stator and the rotor, parameterized by the air gap separation. The graphs correspond to the case of a single chain of SRR in the rotor.

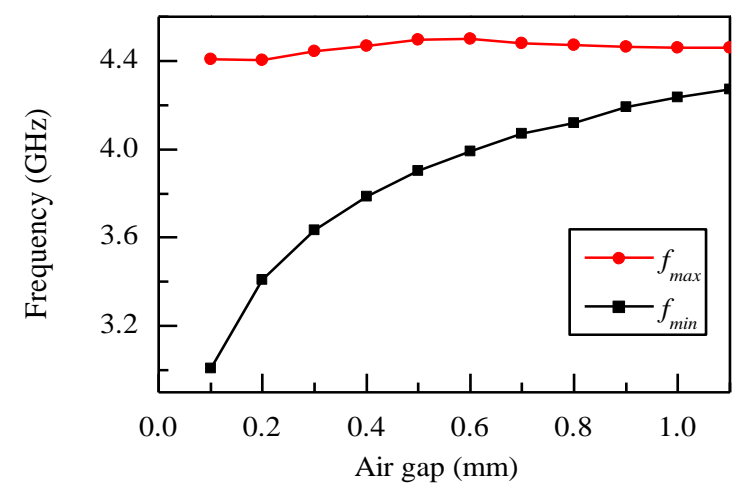

Fig. 12. Upper and lower limit of the frequency range where resonances are present, as a function of the air gap distance.

The constriction of the resonance frequency range as the air gap increases has two negative effects: (i) sensor tuning (i.e., the determination of the signal feeding frequency) is more complex, and (ii) the discrimination of the two attenuation peaks (provided the frequency of the feeding signal is different from the one of the BC-SRR) is degraded (i.e., the attenuation peaks tend to merge). It is important to guarantee that the air gap separation does not experience significant variations during a cycle. If this is not the case, the effect will be a variation of the modulation index with time (see Fig. 13). Moreover, note that if the frequency of the feeding signal is set to the one of the perfectly aligned BC-SRR with the smallest air gap, there will not be attenuation if the gap separation increases. 


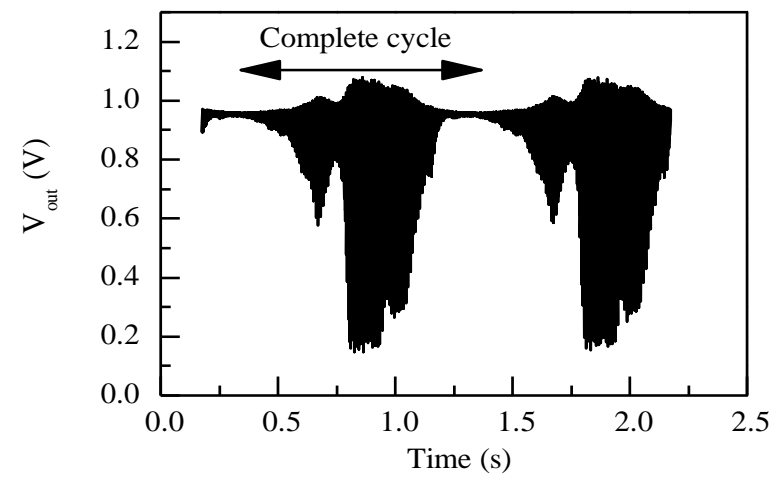

Fig. 13. Measured envelope function over a complete cycle with varying air gap separation.

\section{SENSING System AND MEASUREMENTS}

\section{A. Sensor Schematic and Experimental Set-up}

The schematic of the sensor system, for a rotor with a single chain of SRRs, is represented in Fig. 14. The CPW is fed with a harmonic signal with frequency referred to as carrier frequency, $f_{c}$. As mentioned before, the rotor motion modulates the amplitude of the carrier signal, and from its envelope, the rotation speed can be inferred. To obtain the envelope of the modulated signal, an envelope detector preceded by a circulator is used. The circulator is configured as an isolator (see Fig. 14) and is used to avoid mismatching reflections from the envelope detector. The considered isolator is the ATM ATc4-8, and the envelope detector is implemented by means of the Avago HSMS2860 Schottky diode and the $N 2795 A$ Active probe (which acts as lowpass filter with $R=1 \mathrm{M} \Omega$ and $C=1 \mathrm{pF}$ ), connected to an oscilloscope (the Agilent MSO-X-3104A) in order to visualize the envelope function. The carried signal is generated by means of the Agilent E4438C function generator, whereas the displacement and velocity of the rotor is controlled by the $S T M 23 Q-3 A N$ stepper motor. The experimental set-up is shown in Fig. 15.

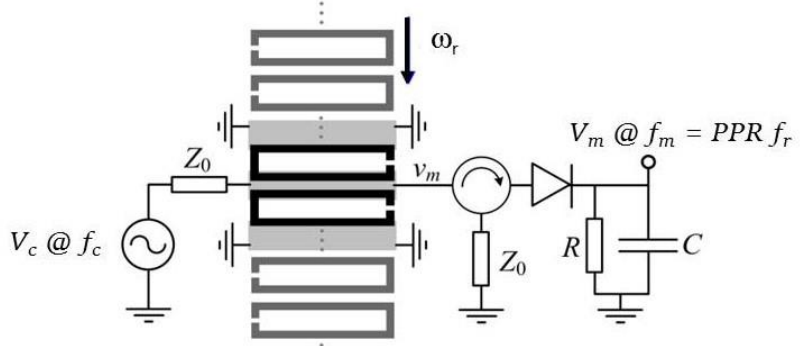

Fig. 14. Schematic for the angular displacement and velocity measurement.

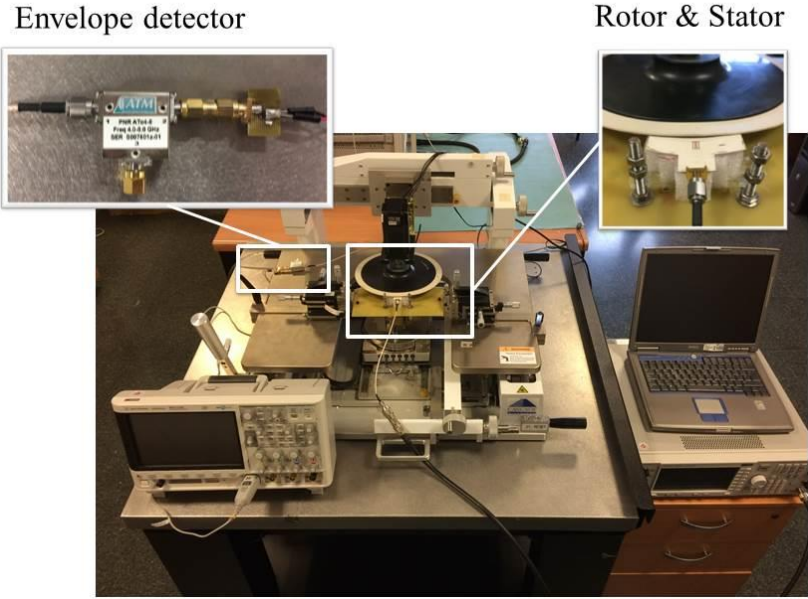

Fig. 15. Experimental set-up including the stepper motor, rotor and stator.

For the measurement of angular velocities, two rotors have been fabricated, one of them with a single circular chain of SRRs, and the other one with two concentric circular SRR chains (Fig. 16). In this figure, the corresponding stators, with a single pair and two pairs of SRRs etched in the back substrate side of the CPW transmission line, are included. The dimensions of the single SRR chain are those indicated in the caption of Fig. 3. For the double-SRR chain, the external SRRs are identical to those of the single chain, whereas the internal SRRs have slightly modified dimensions, in order to accommodate the same number of SRR in both chains (i.e., 300). Such dimensions are indicated in the caption of Fig. 16. The thickness of the rotor substrate, indicated in the caption of Fig. 3, is relatively large in order to provide mechanical robustness to the rotor and to minimize the effects of the rotor assembly on the resonant elements (SRRs) of the chains.

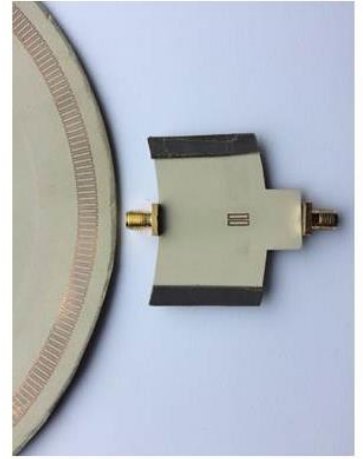

(a)

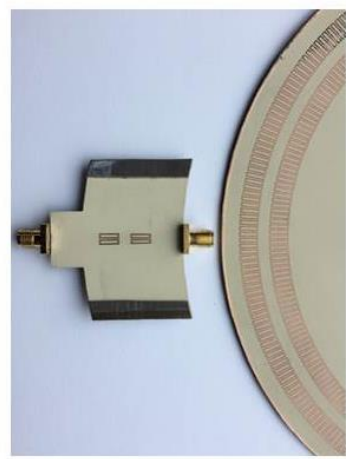

(b)
Fig. 16. Stator and rotor with a single-chain (a) and a double-chain (b) of SRRs. In both cases, the radius of the rotor is $101.6 \mathrm{~mm}$. For the rotor with two SRR chains, SRR dimensions, in reference to Fig. 3, for the inner chain are: $P_{m}=1.81, l_{1}=1.45, l_{2}=6.35, c=0.36$, and $g=0.2$. CPW dimensions are given in the caption of Fig. 3. SRRs have been etched by means of a standard chemical photo-etching technique.

\section{B. Experimental Validation}

The determination of the angular velocity is as follows [39]. The average pulse-to-pulse time distance is

$$
T_{m}=\frac{T_{r}}{P P R},
$$


where $T_{r}$ is the rotation period and $P P R$ is the number of pulses per revolution. The angular speed is derived by measuring $T_{m}$ as follows

$$
\omega_{r}=\frac{d \theta}{d t}=\lim _{\Delta t \rightarrow 0} \frac{\Delta \theta}{\Delta t}=\frac{\theta_{m}}{T_{m}}=\frac{2 \pi / P P R}{T_{m}},
$$

where $\theta$ is the angular position, $t$ is time, and $\theta_{m}$ is the angular period. The angular displacement can then be computed by

$$
\Delta \theta=\omega_{r} \Delta t
$$

The measured transmission coefficients corresponding to Figs. 4 and 8, for the single-chain and double-chain of SRRs rotor, are presented in Fig. 17 (such measurements have been carried out by means of the Agilent PNA N221A vector network analyzer). Note that these results are not in perfect agreement with those inferred from full wave simulation (Figs. 4 and 8). The reason is that the air gap separation is a parameter difficult to accurately control in practice. Nevertheless, the results are very similar, at least the resonance frequencies of the different curves, as indicated by the arrows of Figs. 4 and 8, directly translated to the same positions in Figs. 17(a) and (b). Note that the plots of Figs. 4 and 17(a), and Figs. 8 and 17(b) are drawn to the same scale to ease comparison. The strong resonance for the double SRR chain rotor, corresponding to a misalignment of $\pm P_{m} / 4$ and predicted by the simulation (Fig. 8), is clearly visible in Fig. 17(b).

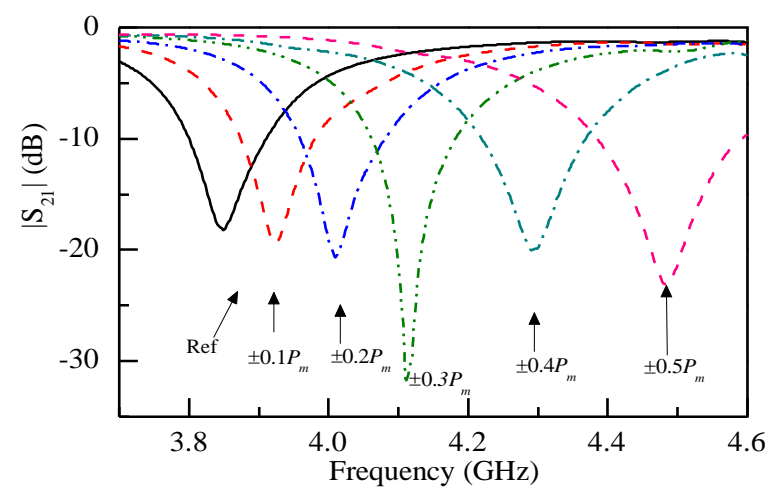

(a)

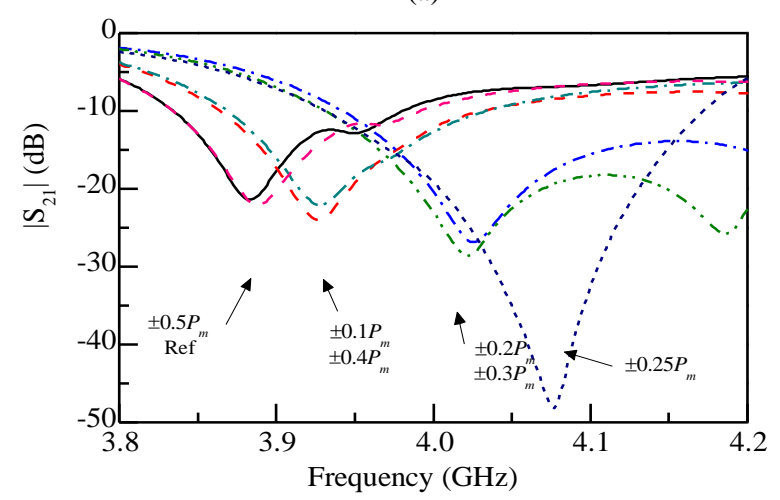

(b)

Fig. 17. Measured transmission coefficient for different relative displacements between the stator and the rotor. (a) Single SRR chain rotor; (b) Double SRR chain rotor.

As mentioned before, it is difficult to precisely control the air gap separation (and hence the minimum resonance frequency, i.e., the one corresponding to the perfectly aligned BC-SRR, $f_{0}$ ). Such controllability is even more complex in a dynamic experiment, i.e., under rotor motion. Therefore, in order to validate the considered scenarios of Section II, with one or two attenuation peaks (or pulses) for the single SRR chain rotor, or with two or four attenuation peaks (or pulses) for the rotor with two SRR chains, we have considered a carrier frequency satisfying $f_{c}>f_{0}$, and we have varied the air gap distance in order to control the number and distance between attenuation peaks, including the possibility of merging (achieved by sufficiently increasing the air gap distance). Interestingly, the time distance between adjacent pulses remains constant, regardless of the number of pulses and carrier frequency. The reason for that is that, according to Figs. 5 and 9, maxima is produced for $P_{m} / 2$ displacement (one pulse), $0 P_{m}$ and $P_{m} / 2$ (two pulses) and $0 P_{m}, P_{m} / 4$ and $P_{m} / 2$ (four pulses), i.e. equidistant space points and, hence, time intervals, if the rotation speed is constant (the number of pulses is relative to the interval $-P_{m} / 2$ up to $+P_{m} / 2$ ). Therefore, such pulseto-pulse time can be considered to compute the rotation speed.

Figure 18 depicts the extracted envelope for a $60-\mathrm{rpm}$ angular velocity, inferred by using the single SRR chain rotor (the carrier frequency was set to $f_{c}=4.20 \mathrm{GHz}$ ). The three curves correspond to different air gap separations. It can be appreciated that, as anticipated, two attenuation peaks (or pulses) appear in general, thus achieving a number of pulses per revolution of $P P R=600$, i.e., twice the number of resonators. However, such attenuation peaks tend to merge as the air gap distance increases. The measured velocity, inferred from the distance between pulses using (2), and indicated in Fig. 18, is in very good agreement with the nominal value $(60 \mathrm{rpm})$ for the different cases (note that the typical tolerance of step motors is 1$2 \%)$.
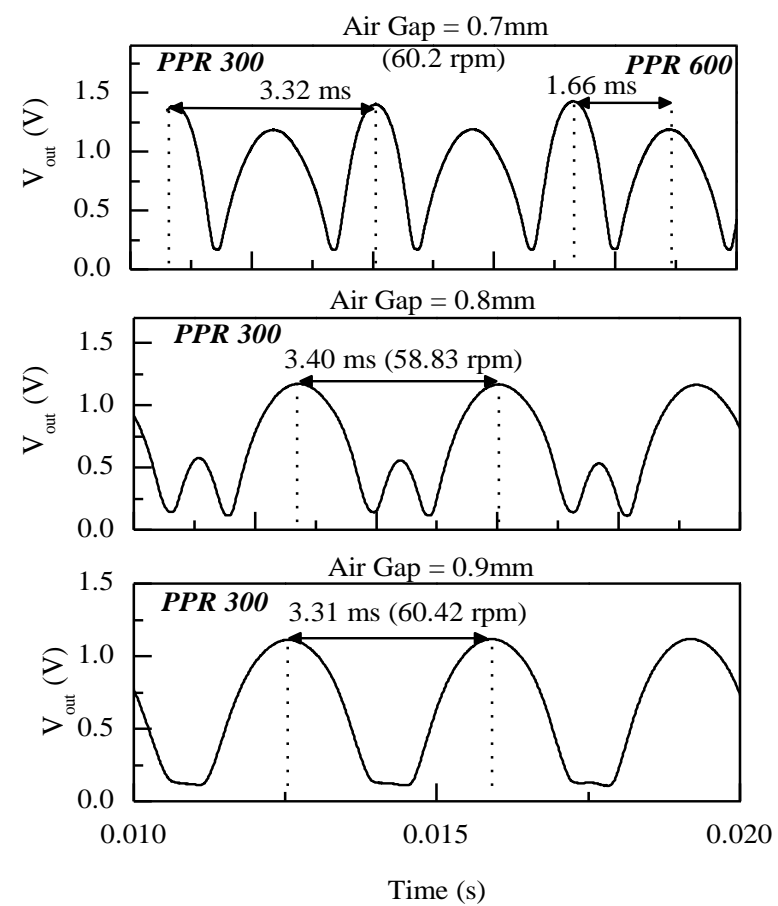

Fig. 18. Envelope of the modulated carrier signal for different air gaps, corresponding to the rotor with a single chain of SRRs. For the smaller air gap, the amplitude of the pulses is similar, and the angular velocity can be obtained from the time distance between adjacent pulses, or between higher amplitude pulses, as indicated. 
We have carried out a similar experiment by replacing the single chain SRR rotor with the rotor with two chains of SRRs. The results, for different air gaps, are depicted in Fig. 19. A similar phenomenology is revealed, but in this case four attenuation peaks for the lower air gap separation are visible, and merging occurs as the gap increases. The measured angular velocity is also in good agreement with the nominal value.

It should be mentioned that for proper measurement of the angular speed, it is necessary to know the number of pulses per revolution. This is 2 and 4, respectively, for the single and the double chain of SRRs rotor, provided the gap is narrow enough. In a robust system, the gap distance can be small and uniform within the rotation cycle (to this end, precession should be avoided); therefore the number of pulses can be a priori known. Sensing with four pulses represents an improvement since it means that the sensor exhibits more angular resolution or, equivalently, more accuracy in the measurement of variations of instantaneous velocities. With the proposed system, practically unlimited angular velocities can be measured since the carrier (feeding) signal frequency is orders of magnitude larger than the angular frequency of any physical (rotating) system.

Determination of the rotation direction (out of the scope of this paper) would be possible by including a third SRR chain with a certain number of resonators representing a code (e.g., presence of resonator corresponding to ' 1 ' and absence to ' 0 '). The sequence of bits (code) would be given by the rotation direction, and hence it would be possible to determine whether rotation is clockwise or counterclockwise.
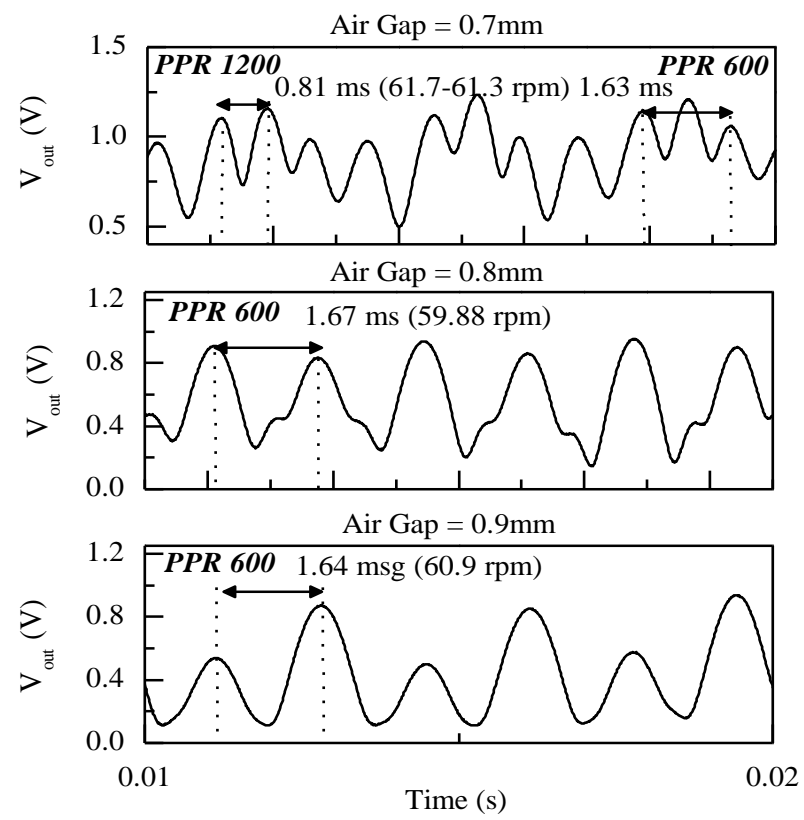

Fig. 19. Envelope of the modulated carrier signal for different air gaps, corresponding to the rotor with two chains of SRRs. The air gap increases from the upper to the lower figure.

Concerning sensor resolution, let us analyze it in more detail. Obviously, angle resolution is given by $P P R$, and it is $1.2^{\circ}, 0.6^{\circ}$ and $0.3^{\circ}$ for the cases with one, two or four pulses per chain period, respectively, corresponding to $P P R$ of 300, 600 and 1200 (note that angular displacement can be inferred from the angular velocity using (3), but it can be also obtained by simply counting up the number of pulses).
The time resolution, $t_{\text {res }}$, is defined as the minimum time interval that can be resolved. Therefore, it is the sampling time interval and it is given by the inverse of the sampling frequency. Time resolution is intimately related to the resolution in the measurement of angular velocities. Namely, if we take into account that the exact distance (perfect time resolution) between adjacent pulses is given by (1), with $t_{\text {res }}$ resolution, we can estimate the rotation frequency as

$$
f_{r} \equiv \frac{1}{T_{r}}=\frac{1}{\left(T_{m} \pm t_{r e s}\right) P P R}
$$

which can be approximated by

$$
f_{r} \approx \frac{1}{P P R \cdot T_{m}}\left(1 \mp \frac{t_{r e s}}{T_{m}}\right)
$$

provided $t_{r e s} \ll T_{m}$. From (5), the resolution in the rotation frequency is

$$
f_{r, r e s}=\frac{t_{r e s}}{P P R \cdot T_{m}^{2}}=P P R \frac{t_{r e s}}{T_{r}^{2}}
$$

As can be seen, the resolution depends on the number of pulses per revolution and rotation speed. For a given rotation speed, the resolution increases (i.e., it is worst) with $P P R$. This is due to the fact that $T_{m}$ decreases. However, by increasing the number of pulses per revolution, variations of instantaneous rotation speed within a revolution can be better detected in time. Note that variations in time lapses smaller than the time between adjacent pulses cannot be detected. If the sampling frequency is $1 \mathrm{MHz}$ (the one in our measurements), then the time resolution is $t_{\text {res }}= \pm 1 \mu \mathrm{s}$. By considering a rotation speed of $60 \mathrm{rpm}$ (corresponding to $T_{r}$ $=1 \mathrm{~s})$ and $P P R=1200$, the resolution in the rotation frequency is found to be $1.2 \mathrm{mHz}$, corresponding to a resolution of $0.072 \mathrm{rpm}$. Note that this very good resolution is achieved due to the high sampling frequency. Therefore, the sampling frequency can be relaxed, yet keeping the resolution within good limits. For a significantly higher rotation speed, i.e. $6000 \mathrm{rpm}$, the resolution is found to be $12 \mathrm{~Hz}$, or $720 \mathrm{rpm}$. In this case, resolution can be improved by simply increasing the sampling frequency (reducing $t_{\text {res }}$ ).

The error in the determination of the instantaneous angular velocity depends on the width of the pulses and distance between them. Narrower pulses provide smaller error. Nevertheless, the accuracy can be improved either by averaging over several pulses, or by considering distant pulses. In both cases, however, the penalty is the degradation in the ability of the sensor to detect speed variations in small time intervals. To give a reasonable (very conservative) estimation of the sensor error, if we consider the width of the pulse as given by the value at $98 \%$ of the pulse maximum, for the double SRR chain rotor and four pulses per chain period (upper Fig. 19), the ratio between the pulse width and the distance between adjacent pulses is 0.07 . This represents a $7 \%$ error in time, but, as mentioned, this value can be reduced by 10 times, i.e., $0.7 \%$ if the considered pulses are separated 10 positions. In terms of error in rotation frequency, these values transform to $7.5 \%$ and $0.705 \%$, respectively.

As compared to optical rotary encoders, the proposed sensors are not competitive in terms of sensor performance. Indeed, a figure of merit in rotary encoders is the number of pulses per revolution, $P P R$, with direct impact on angle resolution and on the capability to detect rotation speed 
variations in short time intervals. Optical encoders can be designed with high values of $P P R$. However, the microwave encoders proposed in this paper are cheaper and can be used in scenarios with harsh environmental conditions, such as space, as an alternative to optical encoders (which may suffer from radiation effects). As compared to other sensors based on potentiometers, aging effects due to wear-out (related to mechanical friction) are avoided, since the proposed sensors are contactless (stator and rotor separated by an air gap).

As compared to other angular velocity sensors based on microwave technology, the sensors proposed in this paper are very competitive in terms of $P P R$. Note, for instance, that the axial sensors proposed in [35]-[37], exhibit only two pulses per revolution (i.e., $P P R=2$ ). Therefore, such axial sensors exhibit very good angular velocity resolution, but such sensors cannot be used to measure instantaneous angular velocities, but just uniform velocities or average values. As angular displacement sensors, the proposed sensors offer competitive angle resolution and are robust to external EMI or noise as compared to the sensors proposed in [33], [35]-[37], based on the variations in the notch depth magnitude. The sensor in [33] offers good sensitivity (variation of the notch depth with the angle), but the dynamic range (for the angle) is very limited. In the proposed sensors, the angle is directly given by the number of pulses so that calibration or linearization is not necessary and the dynamic range is unlimited (since the angle is given by the number of pulses). In [40], the angular displacement and velocity is based on a wireless link between two circularly polarized antennas, and the relative angular displacement between both antennas is determined from the phase inferred from the frequency modulated signal generated in the receiver antenna. This system offers good angle resolution $\left(<1.5^{\circ}\right)$, but velocity is indirectly measured from the angle. Moreover the complete system may be complex in certain applications due to the orientation requirements of the antennas. In our sensor, the angle resolution has been demonstrated to be $0.3^{\circ}$ (best case), angular velocity is directly given by the time distance between pulses, and the system is simple since antennas are not required and it is based on an amplitude modulation scheme.

\section{CONCLUSION}

In conclusion, a detailed analysis of microwave and contactless angular velocity sensors has been carried out in this paper. We have presented the working principle of the sensor, based on the amplitude modulation of a feeding (carrier) signal injected to the stator (a CPW transmission line loaded with at least a pair of split ring resonators, SRRs). It has been shown that this amplitude modulation is caused by the motion of the rotor (a disk of dielectric material containing at least one circular chain of SRRs along its perimeter), and it is due to the coupling between the SRRs of the rotor and those of the stator. It has been shown that by appropriately choosing the frequency of the carrier signal, the number of pulses per revolution (a figure of merit related to sensor accuracy) can be enhanced as compared to previous implementations. Particularly, it has been demonstrated that in single SRR chain rotors, the number of pulses can be twice the number of resonant elements, whereas in rotors with two concentric chains of SRRs (relatively displaced by half a period), it is possible to achieve a number of pulses per revolution up to four times the number of resonators of one of the chains. It has been pointed out that the air gap (or distance between the rotor and stator) plays an important role in sensor performance, and its effects have been analyzed in detail. Finally, we have experimentally validated the analysis carried out by means of an experimental set-up of the sensor system, and two rotors, one with a single chain of SRRs, and the other one with two SRR chains. It has been demonstrated that the phenomenology predicted by simulation, including the effects of the air gap, is verified by experiment. We have pointed out the convenience to consider a working (carrier) frequency above the resonant frequency of the perfectly aligned SRRs of the stator and rotor (due to the difficulty of precisely control the air gap distance), and we have carried out angular velocity measurements with both rotors. The measurements are in good agreement with the nominal values of the considered rotation speeds. The proposed sensors are low cost, as compared to optical rotary encoders, do not suffer from aging effects (due to the absence of mechanical friction), and exhibit a large number of pulses per revolution. The specifications of the considered sensors (carrier frequencies, rotor and stator dimensions, number of pulses per revolution, etc.) are appropriate for applications in reaction wheels (present in space vehicles for attitude control). Thus, conveniently adapted to satisfy space regulations (specific certified materials), the proposed sensors may find practical applications in space environments (not only for measuring the angular displacement/velocity of reaction wheels, but also for the accurate control of pointing mechanisms of antennas, etc).

\section{REFERENCES}

[1] J.B. Pendry, A.J. Holden, D.J. Robbins, and W.J. Stewart, "Magnetism from conductors and enhanced nonlinear phenomena", IEEE Transactions Microwave Theory Techn., vol. 47, pp. 20752084, Nov. 1999.

[2] F. Martín, F. Falcone, J. Bonache, R. Marqués, and M. Sorolla, "Split ring resonator based left handed coplanar waveguide", Appl. Phys. Lett., vol. 83, pp. 4652-4654, Dec. 2003.

[3] F. Falcone, T. Lopetegi, J.D. Baena, R. Marqués, F. Martín, and M. Sorolla, "Effective negative- $\varepsilon$ stop-band microstrip lines based on complementary split ring resonators", IEEE Microw. Compon. Lett., vol. 14, pp. 280-282, Jun. 2004.

[4] F. Falcone, T. Lopetegi, M.A.G. Laso, J.D. Baena, J. Bonache, R. Marqués, F. Martín, and M. Sorolla, "Babinet principle applied to the design of metasurfaces and metamaterials", Phys. Rev. Lett., vol. 93, paper 197401, Nov. 2004.

[5] F. Martín, Artificial Transmission Lines for RF and Microwave Applications, John Wiley, Hoboken, NJ, 2015.

[6] G. Sisó, J. Bonache, M. Gil, and F. Martín, "Application of resonanttype metamaterial transmission lines to the design of enhanced bandwidth components with compact dimensions", Microw. Opt. Techn. Lett., vol. 50, pp. 127-134, Jan. 2008.

[7] J. Bonache, G. Sisó, M. Gil, A. Iniesta, J. García-Rincón, and F. Martín, "Application of composite right/left handed (CRLH) transmission lines based on complementary split ring resonators (CSRRs) to the design of dual band microwave components", IEEE Microw. Compon. Lett., vol. 18, pp. 524-526, Aug. 2008.

[8] M. Durán-Sindreu, G. Sisó, J. Bonache, and F. Martín, "Planar multiband microwave components based on the generalized composite right/left handed transmission line concept", IEEE Trans. Microw. Theory Techn., vol. 58, pp. 3882-3891, Dec. 2010.

[9] G. Zamora, S. Zuffanelli, F. Paredes, F. J. Herraiz Martínez, F. Martín, and J. Bonache, "Fundamental mode leaky-wave antenna (LWA) using slot line and split-ring-resonator (SRR)-based metamaterials", IEEE Antenna Wireless Propag. Lett., vol. 12, pp. 1424-1427, Oct. 2013.

[10] J. Bonache, F. Martín, J. García-García, I. Gil, R. Marqués, and M. Sorolla, "Ultra wide band pass filtres (UWBPF) based on 
complementary split rings resonators", Microw. Opt. Techn. Lett., vol. 46, pp. 283-286, Aug. 2005.

[11] J. Bonache, I. Gil, J. García-García, and F. Martín, "Novel microstrip band pass filters based on complementary split rings resonators", IEEE Trans. Microw. Theory Techn., vol. 54, pp. 265-271, Jan. 2006.

[12] F.J. Herraiz-Martínez, L.E. García-Muñoz, D. González-Ovejero, V. González-Posadas, and D. Segovia-Vargas, "Dual-frequency printed dipole loaded with split ring resonators", IEEE Antenna Wireless Propag. Lett. vol. 8, pp. 137-140, Dec. 2009.

[13] F.J. Herraiz-Martínez, G. Zamora, F. Paredes, F. Martín, and J. Bonache, "Multiband printed monopole antennas loaded with open complementary split ring resonators for PANs and WLANs", IEEE Antenna Wireless Propag. Lett. vol. 10, pp. 1528-1531, Dec. 2011.

[14] J. Naqui, M. Durán-Sindreu, and F. Martín, "On the symmetry properties of coplanar waveguides loaded with symmetric resonators: analysis and potential applications", IEEE MTT-S Int. Microwave Symp. Dig., Montreal (Canada), Jun. 2012.

[15] C. Mandel, B. Kubina, M. Schüßler, and R. Jakoby, "Passive chipless wireless sensor for two-dimensional displacement measurement," in Proc. 41st European Microwave Conf., Manchester, UK, 2011, pp. 79-82.

[16] J. Naqui, M. Durán-Sindreu, and F. Martín, "Novel Sensors Based on the Symmetry Properties of Split Ring Resonators (SRRs)", Sensors vol 11, pp. 7545-7553, Jul. 2011

[17] M. Puentes, C. Weiss, M. Schüßler, and R. Jakoby, "Sensor array based on split ring resonators for analysis of organic tissues," in IEEE MTT-S Int. Microw. Symp., Baltimore, MD, USA, Jun. 2011.

[18] W. Withayachumnankul, K. Jaruwongrungsee, A. Tuantranont, C. Fumeaux, and D. Abbott, "Metamaterial-based microfluidic sensor for dielectric characterization," Sensor Actuat. A Phys., vol. 189, pp. 233-237, Jan. 2013.

[19] A. Ebrahimi, W. Withayachumnankul, S. Al-Sarawi, and D. Abbott, "High-sensitivity metamaterial-inspired sensor for microfluidic dielectric characterization," IEEE Sensors J., vol. 14, no. 5, pp. 1345 1351, May 2014.

[20] A. Abduljabar, D. Rowe, A. Porch, and D. Barrow, "Novel microwave microfluidic sensor using a microstrip split-ring resonator," IEEE Trans. Microw. Theory Techn., vol. 62, no. 3, pp. 679-688, Mar. 2014

[21] A. Ebrahimi, W. Withayachumnankul, S. Al-Sarawi, and D. Abbott, "Metamaterial-inspired rotation sensor with wide dynamic range," IEEE Sensors J., vol. 14, no. 8, pp. 2609-2614, Aug. 2014.

[22] T. Chen, S. Li, and H. Sun, "Metamaterials application in sensing," Sensors, vol. 12, no 3, pp. 2742-2765, Feb. 2012.

[23] T. Chretiennot, D. Dubuc, and K. Grenier, "A microwave and microfluidic planar resonator for efficient and accurate complex permittivitycharacterization of aqueous solutions," IEEE Trans. Microw. Theory Techn., vol. 61, no. 2, pp. 972-978, Feb. 2013.

[24] M. S. Boybay, and O. M. Ramahi, "Material characterization using complementary split-ring resonators," IEEE Trans. Instrum. Measur., vol. 61, no. 11, pp. 3039-3046, Nov. 2012.

[25] A. Ebrahimi, W. Withayachumnankul, S. F. Al-Sarawi, and D. Abbott, "Microwave microfluidic sensor for determination of glucose concentration in water," in 2015 IEEE 15th Mediterranean Microwave Symposium (MMS), Lecce, 2015, pp. 1-3.

[26] A. K. Horestani, J. Naqui, Z. Shaterian, D. Abbott, C. Fumeaux, and F. Martín, "Two-dimensional alignment and displacement sensor based on movable broadside-coupled split ring resonators", Sensors and Actuators A, vol. 210, pp. 18-24, Apr. 2014

[27] J. Naqui, C. Damm, A. Wiens, R. Jakoby, L. Su, J. Mata-Contreras, and F. Martín, "Transmission lines loaded with pairs of stepped impedance resonators: modeling and application to differential permittivity measurements", IEEE Trans. Microw. Theory Techn. vol. 64, no. 11, pp. 3864-3877, Nov. 2016

[28] L. Su, J. Mata-Contreras, J. Naqui, and F. Martín, "Splitter/combiner microstrip sections loaded with pairs of complementary split ring resonators (CSRRs): modeling and optimization for differential sensing applications", IEEE Trans. Microw. Theory Techn., vol 64(12), pp. 4362-4370, Dec. 2016

[29] J. Naqui, M. Durán-Sindreu, and F. Martín, "Alignment and position sensors based on split ring resonators," Sensors, vol. 12, no. 9, pp 11790-11797, Aug. 2012.

[30] J. Naqui, M. Durán-Sindreu, and F. Martín, "Transmission lines loaded with bisymmetric resonators and applications," in IEEE MTT S Int. Microw. Symp., Seattle, WA, USA, Jun. 2013.

[31] J. Naqui and F. Martín, "Mechanically reconfigurable microstrip lines loaded with stepped impedance resonators and potential applications," Int. J. Antennas Propag., vol. 2014, ID 346838, 8 pages, Feb. 2014.
[32] A. Karami-Horestani, C. Fumeaux, S.F. Al-Sarawi, and D. Abbott, "Displacement sensor based on diamond-shaped tapered split ring resonator," IEEE Sensors J., vol. 13, no. 4, pp. 1153-1160, Apr. 2013.

[33] A. Horestani, D. Abbott, and C. Fumeaux, "Rotation sensor based on horn-shaped split ring resonator," IEEE Sens. J., vol. 13, no. 8, pp. 3014-3015, May 2013.

[34] A. K. Horestani, J. Naqui, D. Abbott, C. Fumeaux, and F. Martín, "Two-dimensional displacement and alignment sensor based on reflection coefficients of open microstrip lines loaded with split ring resonators," IET Electron Lett., vol. 50, no. 8, pp. 620-622, Apr. 2014.

[35] J. Naqui and F. Martín, “Transmission lines loaded with bisymmetric resonators and their application to angular displacement and velocity sensors," IEEE Trans. Microw. Theory Techn., vol. 61, no. 12, pp. 4700-4713, Dec. 2013

[36] J. Naqui and F. Martín, "Angular displacement and velocity sensors based on electric-LC (ELC) loaded microstrip lines," IEEE Sensors J., vol. 14, no. 4, pp. 939-940, Apr. 2014.

[37] J. Naqui, J. Coromina, A. Karami-Horestani, C. Fumeaux, and F. Martín, "Angular displacement and velocity sensors based on coplanar waveguides (CPWs) loaded with S-shaped split ring resonator (S-SRR)", Sensors, vol. 15, pp. 9628-9650, Apr. 2015.

[38] D. Schurig, J. J. Mock, and D. R. Smith "Electric-field-coupled resonators for negative permittivity metamaterials." Appl. Phys. Lett., vol. 88, paper 041109, Jan. 2006

[39] J. Naqui and F. Martín, "Application of broadside-coupled split ring resonator (BC-SRR) loaded transmission lines to the design of rotary encoders for space applications", in IEEE MTT-S Int. Microw. Symp. (IMS'16), San Francisco, May 2016.

[40] V. Sipal, A. Z. Narbudowicz, and M. J. Ammann, "Contactless measurement of angular velocity using circularly polarized antennas", IEEE Sensors J., vol. 15, no. 6, pp. 3459-3466, Jun. 2015.

[41] R. Bonert, "Design of a high performance digital tachometer with a microcontroller," IEEE Trans. Instrum. Meas., vol. 38, no. 6, pp. 1104-1108, Dec. 1989

[42] S. J. Arif, M. S. J. Asghar, and A. Sarwar, "Measurement of speed and calibration of tachometers using rotating magnetic field," IEEE Trans. Instrum. Meas., vol. 63, no. 4, pp. 848-858, Apr. 2014.

[43] X. Li, J. Qi, Q. Zhang, and Y. Zhang, "Bias-tunable dual-mode ultraviolet photodetectors for photoelectric tachometer," Appl. Phys. Lett., vol. 104, no. 4, pp. 041108-1-041108-4, Jan. 2014.

[44] E. Shamonina, V.A. Kalinin, K.H. Ringhofer, and L. Solymar, "Magneto-inductive waves in one, two and three dimensions", $J$. Appl. Phys., vol. 92, pp. 6252-6261, Oct. 2002.

[45] R. Marques, F. Medina, and R. Rafii-El-Idrissi, "Role of bianisotropy in negative permeability and left handed metamaterials", Phys. Rev. B, vol. 65, paper 144441, Apr. 2002.

[46] R. Marqués, F. Martín, and M. Sorolla, Metamaterials with Negative parameters: Theory, Design and Microwave Applications, John Wiley, Hoboken, NJ, 2008

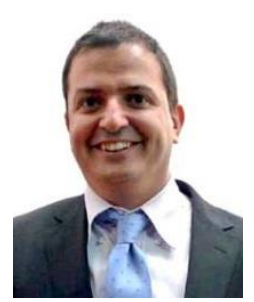

Javier Mata-Contreras was born in 1976 in Málaga (Spain). He received the Ingeniería de Telecomunicación Degree from the Universidad de Málaga (UMA) in 2000 and the $\mathrm{PhD}$ degree from the same university in 2010, with the Thesis "Distributed Amplifiers and Mixers with Transmission Lines based on Metamaterials". In 2000, he joined the UMA Department of Ingeniería de Comunicaciones UMA as Assistant Professor. $\mathrm{He}$ is currently working at CIMITEC and the Universitat Autònoma de Barcelona as Visitant Professor. His research interests include active and passive microwave devices and active distributed circuits based on metamaterials, among others.

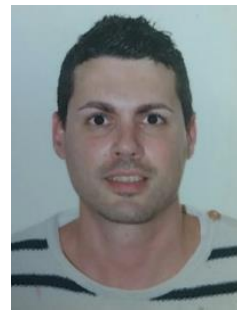

Cristian Herrojo was born in Badalona (Barcelona), Spain, in 1983. He received the Telecommunications Technical Engineering degree (specialty in Electronic Systems) and the Telecommunications Engineering degree in 2010 and 2012, respectively. Currently, he is working toward his $\mathrm{PhD}$ which is mainly focused on the design of RF/microwave resonant structures applied to RFID tags (Radio Frequency Identification) without chip and he has a research grant from FPI Program of the Education and Science Spanish Ministry. 


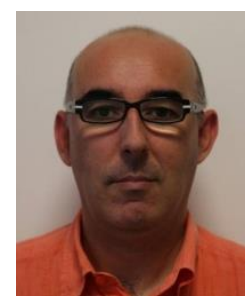

Ferran Martín (M'04-SM'08-F'12) was born in Barakaldo (Vizcaya), Spain in 1965. He received the B.S. Degree in Physics from the Universitat Autònoma de Barcelona (UAB) in 1988 and the $\mathrm{PhD}$ degree in 1992. From 1994 up to 2006 he was Associate Professor in Electronics at the Departament d'Enginyeria Electrònica (Universitat Autònoma de Barcelona), and since 2007 he is Full Professor of Electronics. In recent years, he has been involved in different research activities including modelling and simulation of electron devices for high frequency applications, millimeter wave and $\mathrm{THz}$ generation systems, and the application of electromagnetic bandgaps to microwave and millimeter wave circuits. He is now very active in the field of metamaterials and their application to the miniaturization and optimization of microwave circuits and antennas. He is the head of the Microwave Engineering, Metamaterials and Antennas Group (GEMMA Group) at UAB, and director of CIMITEC, a research Center on Metamaterials supported by TECNIO (Generalitat de Catalunya). He has organized several international events related to metamaterials, including Workshops at the IEEE International Microwave Symposium (years 2005 and 2007) and European Microwave Conference (2009), and the Fifth International Congress on Advanced Electromagnetic Materials in Microwaves and Optics (Metamaterials 2011), where he has acted as chair of the Local Organizing Committee. He has acted as Guest Editor for three Special Issues on Metamaterials in three International Journals. He has authored and co-authored over 450 technical conference, letter, journal papers and book chapters, he is co-author of the book on Metamaterials entitled Metamaterials with Negative Parameters: Theory, Design and Microwave Applications (John Wiley \& Sons Inc.), author of the book Artificial Transmission Lines for RF and Microwave Applications (John Wiley \& Sons Inc.), and he has generated 16 PhDs. Ferran Martín has filed several patents on metamaterials and has headed several Development Contracts.

Prof. Martín is a member of the IEEE Microwave Theory and Techniques Society (IEEE MTT-S). He is reviewer of the IEEE Transactions on Microwave Theory and Techniques and IEEE Microwave and Wireless Components Letters, among many other journals, and he serves as member of the Editorial Board of IET Microwaves, Antennas and Propagation, International Journal of RF and Microwave Computer-Aided Engineering, and Sensors. He is also a member of the Technical Committees of the European Microwave Conference (EuMC) and International Congress on Advanced Electromagnetic Materials in Microwaves and Optics (Metamaterials). Among his distinctions, Ferran Martín has received the 2006 Duran Farell Prize for Technological Research, he holds the Parc de Recerca UAB - Santander Technology Transfer Chair, and he has been the recipient of two ICREA ACADEMIA Awards (calls 2008 and 2013). 